\title{
Structure-Based Drug Design
}

\section{Prashansa Agrawal*}

College of Pharmacy, The University of Arizona, Tucson, Arizona, 85721, USA

In today's world where life is fast-paced, public health is a big concern. With the tremendous ongoing development in the field of pharmaceutical drugs, people can find cure for a vast variety of diseases. These modern-day medicines have been proven to be more effective in treating, managing, controlling and preventing the diseases and their reoccurrence. For example, cancer patients, who had a very low rate of survival in 1970's, can now be treated more efficiently by chemotherapy. At present, the cancer death rate has declined by $20 \%$ in comparison to 1990 [1]. However, along with rapid progress in the efficacy of these pharmaceutical medicines, have come long-term undesirable side effects, poor absorption and clearance, multi-drug resistance, excessive toxicities, and adverse reactions of these medicines. Hence, there is a stringent need to get a closer insight into these drugs in terms of their structure and function, their molecular interactions with large biomolecules (protein, nucleic acids) within the cell, so as to design and/or modify the drugs to have improved potency, fewer side effects and selective binding to their biomolecular target.

Structure-based drug design is a very robust and useful technique. It involves acquisition of the information regarding three-dimensional structure of the molecular target (protein) through methods such as x-ray crystallography, NMR spectroscopy or homology modeling, followed by design of suitable drug candidates based on the binding affinity and selectivity for their target molecules. With the outburst of structural and -omics (e.g. proteomics, genomics, metabolomics) derived data for these protein targets, structure-based drug design has become a very potent and imperative tool for discovering lead compounds which can be optimized to lead towards the development of marketable drug products.

The arena of drug design is disseminating very swiftly. The traditional methods, combinatorial chemistry and high-throughput screening (HTS), for screening hits and developing lead compounds have taken a back seat, as they have not been able to effectively accomplish the goals of discovering new chemical entities or countering the ever-increasing research and development costs for these entities [2]. To overcome these problems, there are now different approaches to design drug based on the structural knowledge of the target and the properties of the ligand.

\section{Structure-Based Drug Design Include}

\section{Fragment-based lead discovery}

This method serves to complement the traditional approaches as a new paradigm in the generation of lead compounds in the process of drug discovery. In this procedure, the leads are identified, optimized, and linked together to produce high-affinity ligands [3] based on the structure-activity relationships (SAR), obtained from NMR [4] and various other techniques, between a small chemical fragment and its protein target. The criteria of selecting the chemical fragments is based on the fact that they should be functionally simpler than drug molecules, have weak potency but efficient binding, have high purity and good aqueous solubility, and follow the 'rule of Three' [5] (molecular weight $<300, \operatorname{Clog} \mathrm{P}<3$, the number of hydrogen bond donors, acceptors and the number of rotatable bonds each should be $<$ 3 ). Then these fragments are expanded or combined to produce a lead with a higher affinity. This method has a plethora of benefits [6] over the conventional high-throughput screening techniques. For example, using this approach, one can find more hydrophilic leads whose affinity can be increased by further addition of hydrophobic groups without allowing the overall lead to become too hydrophobic. In addition, these leads are not expected to contain sterically blocking groups that may interfere with favorable ligand-protein interaction or higher efficiency. The application of fragment-based drug design has gained a lot of attention in recent years. It has lead to the discovery of protein kinase inhibitors [7], matrix metalloproteinase inhibitors [8,9]; site-directed ligand discovery for thymidylate synthase [10] and cysteine aspartyl protease-3 (Caspase-3) [11].

\section{Structure-based virtual screening}

This is a computational approach which involves rapid searching of large libraries of chemical structures in order to identify potential drug candidates (hits) which are most likely to bind to a drug target (protein receptors, enzymes) followed by docking of the hit into a protein target and application of a scoring function to estimate the probability that the drug candidate will bind to the protein target with high affinity [12]. The major advantage of this screening is that it improves the hit rate by considerably decreasing the number of compounds for experimental estimation of their activity and thereby increases the success rate of in vitro experiments. Structure-based virtual screening has been used consistently in pharmaceutical companies and academic groups for early-stage drug discovery.

\section{In silico structure-based lead optimization}

After the desired hits are identified through virtual screening, this method is used to discover and optimize the lead compound from the screened hits based on its high affinity for its target and the elucidation of its absorption, distribution, metabolism, excretion and toxicity (ADMET) properties along with its physicochemical characterization which increases the likelihood for success in clinical phase research. This optimization can be reached using various computational approaches, which include quantitative structure-activity relationships, pharmacophores, similarity search, homology models, databases, data mining, network analysis and data analysis tools. However, like every method, this approach also has its own precincts [13]. The in silico based approach can speed up the search of obtaining the optimized lead by delineating the prediction about its pharmacological properties and thus reducing the in vitro and in vivo experimental time, but sometimes predictions are not accurate due to flexibility of the target,

*Corresponding author: Dr. Prashansa Agrawal, College of Pharmacy, The University of Arizona, Tucson, Arizona, 85721, USA, Tel: +1 520- 626-6749; E-mail: agrawal@pharmacy.arizona.edu

Received June 10, 2013; Accepted June 12, 2013; Published June 14, 2013

Citation: Agrawal P (2013) Structure-Based Drug Design. J Pharmacovigilance 1: e111. doi:10.4172/2329-6887.1000e111

Copyright: (C) 2013 Agrawal P. This is an open-access article distributed under the terms of the Creative Commons Attribution License, which permits unrestricted use, distribution, and reproduction in any medium, provided the original author and source are credited. 
conformational flexibility and non-selectivity of the lead. In such a case, the fragment-based lead discovery strategy can be applied. Hence, all the approaches are complementary to each other.

\section{ADMET modeling}

ADMET stands for absorption, distribution, metabolism, excretion and toxicology of drugs. This method is also referred to as physiologically-based pharmacokinetic modeling. It is used in pharmaceutical research and development, and in health threat evaluation to predict the ADMET of a compound in humans. There are various tools available for envisaging and predicting ADMET data: (1) ADMET parameter predictors, (2) metabolic fate predictors, (3) metabolic stability predictors, (4) cytochrome $\mathrm{P} 450$ substrate predictors and (5) physiology-based pharmacokinetic (PBPK) modeling software [14]. The ADMET parameters influence the performance and pharmacological activity of the compound as a drug based on the kinetics of their exposure to tissues and how they will be treated or accepted by the body, therefore providing a key insight into the nature of a pharmaceutical compound within an organism. This approach plays a crucial role in drug discovery and development as it aids in the selection of compounds during the earliest phases of drug discovery and greatly reduces the late-stage attrition of drugs during pre-clinical / clinical phase trials, consequently proving to be a cost- and timeeffective technique.

At present, even with the advent of numerous above-mentioned pioneering approaches, drug design and development is still an inherently risky business where the input costs are high and the success rate is low. In fact, only a very small percentage of lead compounds ever reach United States Food and Drug Administration (USFDA) approval. Usually, only one in 1,000 lead compounds reaches phase 1 clinical trials and only one in five drugs make it from phase 1 trials into the marketplace [15]. Even for those eventually approved compounds that reach this milestone, there is still $\sim 5 \%$ chance of being removed from the drug development pipeline due to significant adverse drug reactions which enhances mortalities, and at this stage, the cost of failure is extensive. As a result there is a significant quest to develop new experimental or computational tools and scientific approaches to identify correlations between the nature and structure-based properties of the drug and of its safety and efficacy in the human body (pharmacovigilance-based) so that potentially problematic drug leads could be identified at the earliest stages in their development, which will improve the public health and provide a safe, effective and rational use of medicines.

In conclusion, the fusion of structure-based drug design and pharmacovigilance-guided drug design strategies could do wonders in improving the success rate of drug lead optimization, endowing with assistance in monitoring safety and assessing the balance between the hazards and benefits of these drugs either while they are in the development pipeline or have been approved for marketing, reducing the high attrition rates in the clinical phase of the drug development process and decreasing the mortality ratio. In consequence, the structure-based drug design plays a vital role in the field of pharmacovigilance by being complementary to each other in the development of drug discovery.

\section{References}

1. Siegel R, Naishadham D, Jemal A (2013) Cancer statistics, 2013. CA Cancer $\mathrm{J}$ Clin 63: 11-30.

2. Hajduk PJ, Greer J (2007) A decade of fragment-based drug design: strategic advances and lessons learned. Nat Rev Drug Discov 6: 211-219.

3. Bienstock JR (2011) Overview: Fragment-Based Drug Design. Chapter 1, ACS Symposium Series, Vol. 1076:1-26.

4. Shuker SB, Hajduk PJ, Meadows RP, Fesik SW (1996) Discovering high-affinity ligands for proteins: SAR by NMR. Science. 274: 1531-1534.

5. Congreve M, Carr R, Murray C, Jhoti H (2003) A 'rule of three' for fragmentbased lead discovery? Drug Discov Today 8: 876-877.

6. Erlanson DA, McDowell RS, O'Brien T (2004) Fragment-based drug discovery. J Med Chem 47: 3463-3482.

7. Maly DJ, Choong IC, Ellman JA (2000) Combinatorial target-guided ligand assembly: identification of potent subtype-selective c-Src inhibitors. Proc Nat Acad Sci U S A 97: 2419-2424.

8. Hajduk PJ, Seppard G, Nettesheim DG, Olejniczak ET, Shuker SB, et al. (1997) Discovery of Potent Nonpeptide Inhibitors of Stromelysin Using SAR by NMR. J Am Chem Soc 119: 5818-5827.

9. Wada CK, Holms JH, Curtin ML, Dai Y, Florjancic AS, et al. (2002) Phenoxyphenyl sulfone $\mathrm{N}$-formylhydroxylamines (retrohydroxamates) as potent, selective, orally bioavailable matrix metalloproteinase inhibitors. J Med Chem 45: 219-232.

10. Erlanson DA, Braisted AC, Raphael DR, Randal M, Stroud RM, et al. (2000) Site-directed ligand discovery. Proc Natl Acad Sci U S A 97: 9367-9372.

11. Erlanson DA, Lam JW, Wiesmann C, Luong TN, Simmons RL, et al. (2003) In situ assembly of enzyme inhibitors using extended tethering. Nat Biotechnol 21: $308-314$

12. Cheng T, Li Q, Zhou Z, Wang Y, Bryant SH (2012) Structure-based virtual screening for drug discovery: a problem-centric review. AAPS J 14: 133-141.

13. Ekins S, Mestres J, Testa B (2007) In silico pharmacology for drug discovery: applications to targets and beyond. Br J Pharmacol 152: 21-37.

14. Wishart DS (2007) Improving early drug discovery through ADME modelling: an overview. Drugs R D 8: 349-362.

15. Wishart DS (2006) Metabolomics for Drug Discovery, Development and Monitoring. Business Briefing: Future Drug Discovery 1-3. 